\title{
THE REPRESENTATION OF THE MAPPING CLASS GROUP OF A SURFACE ON ITS FUNDAMENTAL GROUP IN STABLE HOMOLOGY
}

\author{
Ulrike Tillmann
}

\begin{abstract}
The natural action of the mapping class group of an orientable or nonorientable surface on its fundamental group induces a group homomorphism into the automorphism group of a free group. In the light of a recent theorem of Galatius [G2], we determine here the map on stable homology.
\end{abstract}

\section{INTRODUCTION AND RESULTS}

Let $\Gamma_{g, 1}$ and $\mathcal{N}_{g, 1}$ be the mapping class groups of an orientable surface $S_{g, 1}$ and a non-orientable surface $N_{g, 1}$ of genus $g>1$ with one boundary component. The underlying diffeomorphisms are assumed to fix the boundary point-wise. Indeed, there are well-known homotopy equivalences [EE], [ES]

$$
\begin{aligned}
& \pi_{0}: \operatorname{Diff}^{+}\left(S_{g, 1} ; \partial\right) \stackrel{\simeq}{\longrightarrow} \Gamma_{g, 1} ; \\
& \pi_{0}: \operatorname{Diff}\left(N_{g, 1} ; \partial\right) \stackrel{\simeq}{\longrightarrow} \mathcal{N}_{g, 1} .
\end{aligned}
$$

Note that $S_{g, 1}$ and $N_{g, 1}$ are homotopic to a wedge of $2 g$ and $g$ circles. The action of the mapping class groups on the fundamental groups of the surfaces (with a base-point on the boundary) thus induce representations

$$
\rho^{+}: \Gamma_{g, 1} \longrightarrow \text { Aut }_{2 g} \text { and } \rho: \mathcal{N}_{g, 1} \longrightarrow \text { Aut F }_{g}
$$

where $\mathrm{F}_{n}$ denotes the free group on $n$ generators. These representations are covered by maps of $\mathrm{H}$-spaces that send a diffeomorphism to the homotopy equivalence it defines. Indeed, the $\mathrm{H}$-space of base-point preserving homotopy equivalences of a wedge of $n$ circles is homotopy equivalent to its group of connected components, Aut $\mathrm{F}_{n}$.

Both mapping class groups can be stabilized with respect to the genus by sewing a torus or a projective plane with two boundary components to $S_{g, 1}$ or $N_{g, 1}$ and extending diffeomorphisms by the identity. Similarly, the automorphism groups of free groups can be stabilized with respect to the number of generators in the free group. The two representations are compatible with these stabilizations. Recall

1991 Mathematics Subject Classification. Primary 20F34, 20F29; Secondary 57N05, 55P47. 
from $[\mathrm{H}]$ and [I1], [W2], and [HV] respectively, that the natural inclusions induce homology isomorphisms (with any constant coefficient system) in the stable range:

$$
\begin{aligned}
H_{*}\left(\Gamma_{g, 1}\right) \stackrel{\simeq}{\longrightarrow} H_{*}\left(\Gamma_{\infty}\right) & \text { for } g \geq 2 *+1, \\
H_{*}\left(\mathcal{N}_{g, 1}\right) \stackrel{\simeq}{\longrightarrow} H_{*}\left(\mathcal{N}_{\infty}\right) & \text { for } g \geq 4 *+3, \\
H_{*}\left(\text { Aut }_{n}\right) \stackrel{\simeq}{\longrightarrow} H_{*}\left(\text { Aut }_{\infty}\right) & \text { for } n \geq 2 *+2 .
\end{aligned}
$$

In homological terms our main results can be stated as follows.

Theorem 1. $\rho^{+}: \Gamma_{g, 1} \rightarrow A u t F_{2 g}$ induces a split surjection on homology with $\mathbb{Z}[1 / 2]$-coefficients, and is a surjection onto $2 H_{*}\left(\right.$ Aut $\left.F_{2 g} ; \mathbb{Z}_{(2)}\right)$ for $g \geq 2 *+1$.

Theorem 2. $\rho: \mathcal{N}_{g, 1} \rightarrow$ Aut $F_{g}$ induces a split surjection in homology with any constant coefficient system for $g \geq 4 *+3$.

For the symmetric groups $\Sigma_{n}$ the stable homology range is $n \geq 2 *$, cf. [N]. $\Sigma_{n}$ acts on $\mathrm{F}_{n}$ by permutation of the generators. This defines a group homomorphism incl : $\Sigma_{n} \hookrightarrow$ Aut $_{n}$. Galatius proves in [G2]:

$$
H_{*}\left(\Sigma_{n}\right) \stackrel{\simeq}{\longrightarrow} H_{*}\left(\text { Aut }_{n}\right) \quad \text { for } n \geq 2 *+2 \text {. }
$$

Thus, in as far the stable homology of the symmetric group is known, so are the images of $\rho^{+}$and $\rho$. In particular, this includes the homology with field coefficients.

Both theorems, variants of which we discuss in the next section, are implied by their stronger homotopy theoretic versions, Theorem 5 and Theorem 6 below. These in turn follow immediately from the main theorem in [G2] once it is shown that the representations $\rho^{+}$and $\rho$ induce maps of infinite loop space. For the mapping class groups of orientable surfaces the latter was proved by Wahl [W1]. Relying on [G2], we give a short argument for this that immediately can be extended to the non-orientable case as well.

\section{VARIANTS OF THE MAIN THEOREMS}

Before we turn to the proof of the main theorems we discuss in this section a few corollaries and remarks concerning mapping class groups of other surfaces.

2.1. Decorated surfaces. Consider the oriented surface $S_{g, n+1}^{k}$ with $n+1$ (parametrised) boundary components and $k$ marked points. Let $\Gamma_{g, n+1}^{k}$ be the mapping class group associated to the diffeomorphisms of $S_{g, n+1}^{k}$ that fix all boundary and marked points, and let $\Gamma_{g,(n), 1}^{(k)}$ be associated to its diffeomorphisms that may permute the marked points and $n$ of the boundary components while preserving their parametrisation; one boundary component is point-wise fixed to allow stabilization. Representations on the fundamental group of the underlying surfaces give 
rise to a commutative diagram

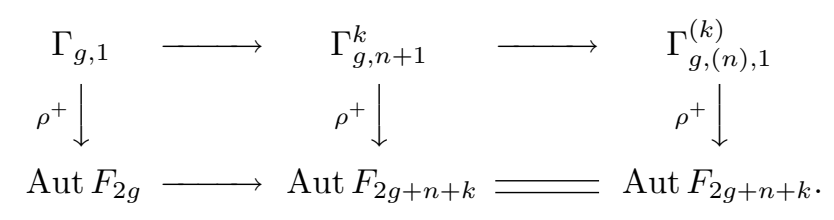

The horizontal map on the bottom is an isomorphism in homology in degrees with $g \geq *+1$. Thus it follows immediately that Theorem 1 is also valid for the representations

$$
\rho^{+}: \Gamma_{g, n+1}^{k} \longrightarrow \text { Aut } F_{2 g+n+k} \quad \text { and } \quad \rho^{+}: \Gamma_{g,(n), 1}^{(k)} \longrightarrow \text { Aut } F_{2 g+n+k} .
$$

A similar argument shows that Theorem 2 holds for the decorated non-orientable mapping class groups and their representations

$$
\rho: \mathcal{N}_{g, n+1}^{k} \longrightarrow \text { Aut } F_{g+n+k} \quad \text { and } \quad \rho^{+}: \mathcal{N}_{g,(n), 1}^{(k)} \longrightarrow \text { Aut } F_{g+n+k}
$$

2.2. Braid groups. The ranges of homology degrees for the results established in 2.1 depend only on the genus $g$. One might hope to gain more information in the decorated case with increasing $k$ or $n$. We offer the following remarks.

The representation of the braid group $\mathrm{Br}_{k}=\Gamma_{0,1}^{(k)}$ on the first homotopy group of the underlying surface is just Artin's inclusion map

$$
\phi: \mathrm{Br}_{k} \longrightarrow \text { Aut } F_{k}
$$

Recall from [T3] that in stable homology with field coefficients $\mathbb{Q}$ or $\mathbb{Z} / p \mathbb{Z}$, this map is trivial unless $p=2$, in which case it is an inclusion. More precisely, integrally the image is precisely the image of the map induced by the canonical map of groups $\mathrm{Br}_{k} \rightarrow \Sigma_{k}$ followed by incl $: \Sigma_{k} \rightarrow$ Aut $F_{k}$. From the commutative diagram of groups

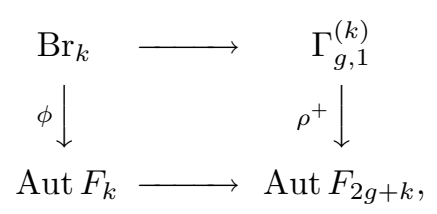

we see that also the image of $\rho^{+}$contains $\operatorname{Im}\left(H_{*}\left(\operatorname{Br}_{k}\right) \rightarrow H_{*}\left(\Sigma_{k}\right)\right)$ in the stable range, and in particular the non-trivial element in $H_{1}\left(\right.$ Aut $\left.F_{2 g+k}\right)=\mathbb{Z} / 2 \mathbb{Z}$ for $k \geq 4$. Thus as $k$ increases additional 2 -torsion classes are detected in the homology of the automorphism group of a free group.

When surfaces with additional boundary components are considered, the braid group $\mathrm{Br}_{k}$ is replaced by the ribbon braid group $\mathrm{RBr}_{k}$. But the representation of $\Gamma_{g,(n), 1}$ factors through $\Gamma_{g, 1}^{(n)}$ as the fundamental group of the underlying surfaces does not see the difference between a boundary component or a puncture. Hence the analysis reduces to that just given above.

These remarks also carry over to the non-orientable case. 
2.3. Once punctured surfaces. The mapping class groups of once punctured surfaces only give rise to outer automorphisms of the fundamental groups of the underlying surfaces as no point of the surface is fixed by all diffeomorphisms:

$$
\rho^{+}: \Gamma_{g}^{1} \longrightarrow \text { Out }_{2 g} \quad \text { and } \quad \rho: \mathcal{N}_{g}^{1} \longrightarrow \text { Out }_{g}
$$

Consider the commutative diagrams

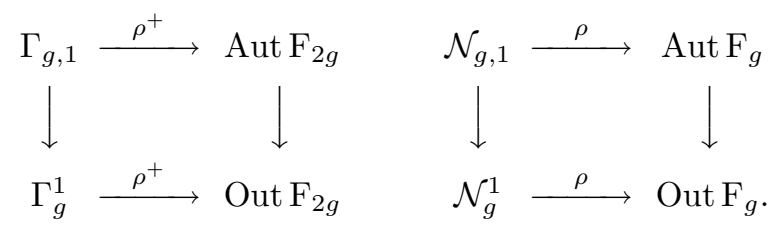

By [HV] the natural map Aut $\mathrm{F}_{n} \rightarrow \mathrm{OutF}_{n}$ induces a homology isomorphism in degrees $*$ for $n \geq 2 *+4$. Hence Theorem 1 and 2 also hold for the maps in (2.1) in the same ranges, $g \geq 2 *+1$ and $g \geq 4 *+3$ respectively.

We note that this range is slightly better then that which would be trivially implied by the fact that the maps $\Gamma_{g, 1} \rightarrow \Gamma_{g}^{1}$ and $\mathcal{N}_{g, 1} \rightarrow \mathcal{N}_{g}^{1}$ induce inclusions in homology in degrees $*$ for $g \geq 2 *+2$, cf. [I2], and $g \geq 4 *+5$, cf. [W2].

2.4. Closed surfaces. The mapping class group of the closed surface $S_{g}$ maps to the outer automorphisms group of the surface group $\pi_{1} F_{g}$, and not a free group. By classical theorems of Nielsen and Bers, we know that

$$
\rho^{+}: \Gamma_{g} \longrightarrow \operatorname{Out}\left(\pi_{1} F_{g}\right)
$$

is an isomorphism onto the index two, normal subgroup of orientation preserving outer automorphisms.

The situation for non-orientable surfaces is analogous, and the representation

$$
\rho: \mathcal{N}_{g} \longrightarrow \operatorname{Out}\left(\pi_{1} N_{g}\right)
$$

is an isomorphism.

\section{The $A$-ThEORY TRANSFER MAP}

We recall briefly the construction of the map of infinite loop spaces from $\mathbb{Z} \times$ $B \Gamma_{\infty}^{+}$to Waldhausen $K$-theory of a point $A(*)$ from [T2] and note that the same construction gives a map of infinite loop spaces from $\mathbb{Z} \times B \mathcal{N}_{\infty}^{+}$to $A(*)$. Indeed, we will construct both maps simultaneously.

Let $\mathcal{C}^{ \pm}$be the 2-category of oriented and non-orientable surfaces. Its objects are the natural numbers where $n \geq 0$ represents $n$ copies of the standard circle. Its 1-morphisms are generated under disjoint union and sewing by the following atomic surfaces, each equipped with a fixed parameterisation of its boundary components and an orientation if one exists: a disk $D \in \mathcal{C}^{ \pm}(0,1)$, a torus with two 
boundary components $T \in \mathcal{C}^{ \pm}(1,1)$, a projective plane with two boundary components $R \in \mathcal{C}^{ \pm}$, and a pair of pants $P \in \mathcal{C}^{ \pm}(2,1)$. Furthermore, we add elements of the symmetric group represented by cylinders of length 0 . The set of 2-morphisms between two surfaces $F$ and $F^{\prime}$ is the topological space $\operatorname{Diff}\left(F, F^{\prime} ; \partial\right)$ of all diffeomorphisms from $F$ to $F^{\prime}$ that preserve the boundary parameterisation and labelling, as well as the orientation if one exists.

We consider two subcategories of $\mathcal{C}^{ \pm}$. Let $\mathcal{C}^{+}$be the subcategory which contains only oriented surfaces and orientation preserving diffeomorphisms, and let $\mathcal{C}^{-}$be the subcategory that contains no orientable surfaces of positive genus. Using Harer's homology stability theorem for the oriented mapping class groups and Wahl's homology stability theorem for the non-oriented mapping class groups, the group completion theorem for categories gives the following equivalences, cf. [T1], [W2]:

$$
\begin{aligned}
\Omega B \mathcal{C}^{+} & \simeq \mathbb{Z} \times B \Gamma_{\infty}^{+} \\
\Omega B \mathcal{C}^{-} \simeq \Omega B \mathcal{C}^{ \pm} & \simeq \mathbb{Z} \times B \mathcal{N}_{\infty}^{+} .
\end{aligned}
$$

Disjoint union induces a symmetric monoidal structure on $\mathcal{C}^{ \pm}$and its subcategories $\mathcal{C}^{-}$and $\mathcal{C}^{+}$, and therefore defines infinite loop space structures on the associated classifying spaces.

Identify $A(*)$ with the loop space of $|w \cdot S(*)|$ where $w \cdot S(*)$ is Waldhausen's simplicial set construction applied to the category of finite spaces over a point $*$. A $k$-simplex is a $k$-flag of cofibrations over $*$. We can map the nerve $N . \mathcal{C}^{ \pm}$to $w . S(*)$ by sending a $k$-simplex of composable surfaces $\left(F_{1}, \ldots, F_{k}\right)$ to the cofibration

$$
* \hookrightarrow F_{1} / \partial_{0} \hookrightarrow F_{1} \circ F_{2} / \partial_{0} \hookrightarrow \cdots \hookrightarrow F_{1} \circ \cdots \circ F_{k} / \partial_{0} .
$$

Here $\circ$ denotes composition of surfaces under sewing, and $F / \partial_{0}$ is the quotient space of $F$ in which the source boundary $\partial_{0}$ is collapsed to a point; if the incoming boundary is empty, a disjoint base-point is added. Diffeomorphisms clearly induce based weak equivalences. As disjoint union is taken to the wedge product, this construction induces maps of infinite loop spaces.

Proposition 3. $\chi^{+}: \mathbb{Z} \times B \Gamma_{\infty}^{+} \rightarrow A(*)$ and $\chi: \mathbb{Z} \times B \mathcal{N}_{\infty}^{+} \rightarrow A(*)$ are maps of infinite loop spaces.

\section{The REPRESENTATIONS AS INFINITE LOOP MAPS}

We now deduce from the main result of [G2] and Waldhausen's splitting theorem that the representations $\rho^{+}$and $\rho$ induce maps of infinite loop spaces.

For any category $\mathcal{D}$, an element in the space of morphisms $\mathcal{D}(a, b)$ from an object $a$ to an object $b$ defines a path from $a$ to $b$ in the classifying space $B \mathcal{D}$. Extending this construction to 2-categories defines the maps labelled by $\mathrm{c}$ in the commutative 
diagram below:

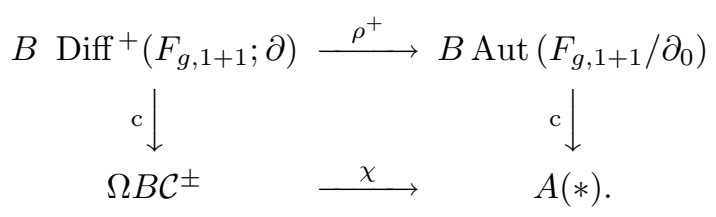

Here Aut $(X)$ for a based space $X$ denotes the space of based homotopy equivalences of $X$. Note that $F_{g, 1+1} / \partial_{0}$ is a wedge of $2 g$ or $g$ circles depending on whether $F_{g, 1+1}$ is orientable or not. The vertical maps $\mathrm{c}$ extend over the stabilisation with respect to the genus and over the plus construction. Thus $\chi^{+}$and $\chi$ factor through $\rho^{+}$and $\rho$ respectively. Recall from $[\mathrm{W}]$ that the composition of the maps

$$
\mathbb{Z} \times B \Sigma_{\infty}^{+} \stackrel{\text { incl }}{\longrightarrow} \mathbb{Z} \times B \text { Aut }{ }_{\infty}^{+} \stackrel{c}{\longrightarrow} A(*) \stackrel{t r}{\longrightarrow} Q S^{0}
$$

is a homotopy equivalence of infinite loop spaces; here $t r$ denotes Waldhausen's trace map. By the main result of [G2] (see 1.2 here), also the first map is a homotopy equivalence. Hence, up to a self-homotopy equivalence of $Q S^{0}$ as infinite loop space,

$$
\rho^{+} \sim \operatorname{tr} \circ \chi^{+} \text {and } \rho \sim t r \circ \chi
$$

$t r$ is a map of infinite loop spaces, and by Proposition 3 so are $\chi^{+}$and $\chi$. Hence, $\rho^{+}$and $\rho$ are maps of infinite loop spaces. This extends Wahl's result [W1] to the mapping class group of non-orientable surfaces.

Theorem 4. $\rho^{+}: \mathbb{Z} \times B \Gamma_{\infty}^{+} \rightarrow \mathbb{Z} \times B A u t_{\infty}^{+}$and $\rho: \mathbb{Z} \times B \mathcal{N}_{\infty}^{+} \rightarrow \mathbb{Z} \times B$ Aut $t_{\infty}^{+}$are maps of infinite loop spaces.

\section{THE MAIN THEOREMS AND A COUPLE MORE REMARKS}

To state our results we will make use of the notation $X_{(p)}$ for the localisation of a space $X$ at a prime $p$. Recall that a connected component of $Q S^{0}$ splits as a product of its $p$-localisations. Thus we have

$$
B \Sigma_{\infty}^{+} \simeq B \mathrm{Aut}_{\infty}^{+} \simeq Q_{0} S^{0} \simeq \prod_{p} Q_{0} S_{(p)}^{0}
$$

Theorem 5. There exists a map of infinite loop spaces $\sigma^{+}: Q S^{0} \rightarrow \mathbb{Z} \times B \Gamma_{\infty}^{+}$such that the composition with $\rho^{+}: \mathbb{Z} \times B \Gamma_{\infty}^{+} \rightarrow \mathbb{Z} \times B$ Aut $t_{\infty}^{+} \simeq Q S^{0}$ restricted to $Q S_{(p)}^{0}$ is homotopic to the identity for all odd primes $p$, and the loop sum doubling map for $p=2$. 
Theorem 6. There exists a map of infinite loop spaces $\sigma: Q S^{0} \rightarrow \mathbb{Z} \times B \mathcal{N}_{\infty}^{+}$such that the composition with $\rho: \mathbb{Z} \times B \mathcal{N}_{\infty}^{+} \rightarrow \mathbb{Z} \times B$ Aut $t_{\infty}^{+} \simeq Q S^{0}$ is homotopic to the identity.

These two theorems imply immediately Theorem 1 and 2 from the introduction. The stated range of homology surjectivity is the minimum for the relevant ranges for homology isomorphisms listed in (1.1) and (1.2). We will prove both Theorem 5 and 6 at the same time.

Proof: Consider the following compositions:

$$
\begin{aligned}
& Q S^{0} \stackrel{\sigma^{+}}{\longrightarrow} \mathbb{Z} \times B \Gamma_{\infty}^{+} \stackrel{\rho^{+}}{\longrightarrow} Q S^{0} \\
& Q S^{0} \stackrel{\sigma}{\longrightarrow} \mathbb{Z} \times B \mathcal{N}_{\infty}^{+} \stackrel{\rho}{\longrightarrow} Q S^{0} .
\end{aligned}
$$

Based maps of infinite loop spaces from $Q S^{0}$ are determined by their restriction to $S^{0}$. The maps $\sigma^{+}$and $\sigma$ are defined to be the based infinite loop maps that send the non-base-point in $S^{0}$ to $(1, *)$ in $\mathbb{Z} \times B \Gamma_{\infty}^{+}$and $\mathbb{Z} \times B \mathcal{N}_{\infty}^{+}$respectively. As $\rho^{+}$ and $\rho$ are maps of infinite loop spaces, so are the compositions $\rho^{+} \sigma^{+}$and $\rho \sigma$. It easy to see that on components the representations are the times 2 map and the identity respectively,

$$
\rho^{+}(1, *)=(2, *) \quad \text { and } \quad \rho(1, *)=(1, *) .
$$

Thus the composed maps in (5.1) are the self-maps [2] and [1] of $Q S^{0}$, and $\sigma^{+}$ restricted to the $p$-localisation for odd primes $p$, and $\sigma$ are the required splitting maps.

5.1. Homology representations. Instead of considering the representation on the first homotopy groups of the underlying surfaces we may consider the representation on the first homology groups

$$
h^{+}: \Gamma_{g, 1} \longrightarrow \mathrm{GL}_{2 g} \mathbb{Z} \quad \text { and } \quad h: \mathcal{N}_{g, 1} \longrightarrow \mathrm{GL}_{g} \mathbb{Z} .
$$

They factor through $\rho^{+}$and $\rho$, and after stabilisation, taking classifying space and applying the plus construction, they factor through

$$
Q S^{0} \simeq \mathbb{Z} \times B \mathrm{Aut}_{\infty}^{+} \longrightarrow A(*) \stackrel{L}{\longrightarrow} K(\mathbb{Z})
$$

where $L$ is the linearisation map from Waldhausen $A$-theory to algebraic $K$-theory. The map (5.2) has been studied in [Q]. In particular, for $p$ odd and $q$ a unit in $\mathbb{Z} / p^{2} \mathbb{Z}$, the composition with the natural map to $K(\mathbb{Z} / q \mathbb{Z})_{(p)} \simeq \operatorname{Im} J_{(p)}$ is a split surjection. So $h^{+}$and $h$ satisfy Theorem 5 and 6 with $Q S^{0}$ replaced by $\operatorname{Im} J_{(1 / 2)}$, the image of $J$ localised away from 2 . Note though that in this case the splitting is not a map of infinite loop spaces. In the oriented case, this splitting of $\mathbb{Z} \times B \Gamma_{\infty}^{+}$was previously proved by Charney and Cohen [CC]. It is easy to see that the representations $h^{+}$ and $h$ induce maps of infinite loop spaces. Hence, our analogue of Theorem 5 and 6 for the homology representations does not depend on [G2]. 
5.2. Representations as transfer maps. The stable homology of both $\Gamma_{\infty}$ and $\mathcal{N}_{\infty}$ are well understood because by [MW] and [W2] we have

$$
\mathbb{Z} \times B \Gamma_{\infty}^{+} \simeq \Omega^{\infty} M T S O_{2} \quad \text { and } \quad \mathbb{Z} \times B \mathcal{N}_{\infty}^{+} \simeq \Omega^{\infty} M T O_{2}
$$

and the homology groups with coefficients in $\mathbb{F}_{p}$ and $\mathbb{Q}$ of the right hand spaces are known, cf. [G1] and $[\mathrm{R}-\mathrm{W}]$. We also recall here that there are natural maps

$$
\omega^{+}: \Omega^{\infty} \mathrm{MTSO}_{2} \longrightarrow Q\left(\mathrm{BSO}(2)_{+}\right) \quad \text { and } \quad \omega: \Omega^{\infty} \mathrm{MTO}_{2} \longrightarrow Q\left(\mathrm{BO}(2)_{+}\right)
$$

and split projections $p r: Q\left(X_{+}\right) \rightarrow Q S^{0}$ for any space $X$ with an adjoint basepoint. Douglas proves in $[\mathrm{D}]$ that the $A$-theory transfer composed with the trace map is homotopic to the Becker-Gottlieb transfer map. It follows immediately from the definitions of $\omega^{+}$and $\omega$ that stably we have the following interpretation of the representations:

$$
\rho^{+} \simeq[-2] \circ p r \circ \omega^{+} \text {and } \quad \rho \simeq[-1] \circ p r \circ \omega
$$

\section{REFERENCES}

[CC] R. Charney, F. R. Cohen, A stable splitting for the mapping class group, Michigan Math. J. 35 (1988), 269-284.

[D] C.L. Douglas, Trace and transfer maps in the algebraic K-theory of spaces, $K$-Theory 36 (2005), 59-82.

[EE] C.J. Earle, J. Eells, A fibre bundle description of Teichmüller theory, J. Diff. Geom. 3 (1969), 19-43.

[ES] C.J. Earle, A. Schatz, Teichmüller theory for surfaces with boundary, J. Diff. Geom. 4 (1970), 169-185.

[G1] S. Galatius, Mod p homology of the stable mapping class group, Topology 43 (2004), 11051132.

[G2] S. Galatius, Stable homology of automorphism groups of free groups, math.AT/0610216.

$[\mathrm{H}]$ J.L. Harer, Stability of the homology of the mapping class groups of orientable surfaces, Annals Math. 121 (1985), 215-249.

[HV] A. Hatcher, K. Vogtmann, Homology stability for outer automorphism groups of free groups, Algebr. Geom. Topol. 4 (2004), 1253-1272.

[I1] N.V. Ivanov, Stabilization of the homology of Teichmüller modular groups, Original: Algebra i Analiz 1 (1989), 110-126; Translated: Leningrad Math. J. 1 (1990), 675-691.

[I2] N.V. Ivanov, On the homology stability for Teichmüller modular groups: closed surfaces and twisted coefficients, Mapping class groups and moduli spaces of Riemann surfaces (Göttingen, 1991/Seattle, WA, 1991), Contemp. Math., vol. 150, Amer. Math. Soc., Providence RI, 1993, pp. 149-194.

[MW] I. Madsen, M. Weiss, The stable moduli space of Riemann surfaces: Mumford's conjecture, Ann. of Math. 165 (2007), 843-941.

[N] M. Nakaoka, Decomposition theorem for homology groups of symmetric groups, Ann. of Math. 71 (1960), 16-42.

[Q] D. Quillen, Letter from Quillen to Milnor on $\operatorname{Im}\left(\pi_{i} 0 \rightarrow \pi_{i}{ }^{\mathrm{S}} \rightarrow K_{i} \mathbb{Z}\right)$., Algebraic $K$-theory (Proc. Conf., Northwestern Univ., Evanston, Ill., 1976) Lecture Notes in Math., Springer, Berlin, vol. 551, 1976., pp. 182-188.

[R-W] O. Randal-Williams, The homology of the stable non-orientable mapping class group, Alg. Geom. Top. 8 (2008), 1811-1832.

[T1] U. Tillmann, On the homotopy of the stable mapping class group, Invent. Math. 130 (1997), 257-275. 
[T2] U. Tillmann, A splitting for the stable mapping class group, Math. Proc. Cam. Phil. Soc. 127 (1999), 55-65.

[T3] U. Tillmann, Artin's map in stable homology, Bull. London Math. Soc. 39 (2007), 989-992.

[W1] N. Wahl, From mapping class groups to automorphism groups of free groups, J. London Math. Soc. 72 (2005), 510-524.

[W2] N. Wahl, Homological stability for the mapping class groups of non-orientable surfaces, Invent. Math. 171 (2008), 389-424.

[W] F. Waldhausen, Algebraic K-theory of topological spaces II, Algebraic topology, Aarhus 1978, Springer LNM, 1979, pp. 356-394.

Mathematical Institute, Oxford University, 24-29 St Giles St., Oxford OX1 3LB, $\mathrm{UK}$

E-mail address: tillmann@maths.ox.ac.uk 